\title{
Dynamics of Executive Control and Motor Deficits in Parkinsonian Rats
}

\author{
Alain Courtière, ${ }^{1}$ Jeanine Hardouin, ${ }^{2}$ Borís Burle, ${ }^{3}$ Franck Vidal, ${ }^{3}$ Nathalie Turle-Lorenzo, ${ }^{3}$ Marianne Amalric, ${ }^{3}$ \\ and Thierry Hasbroucq ${ }^{3}$ \\ ${ }^{1}$ Institut de Recherche Biomédicale des Armées, 91223 Brétigny-sur-Orge, France, ${ }^{2}$ Institut de Recherche Biomédicale des Armées, 83041 Toulon, France, \\ ${ }^{3}$ Centre National de la Recherche Scientifique, and Aix-Marseille Université, UMR 6155, 13331 Marseille, France
}

While there is general agreement that in Parkinson's disease (PD), striatal dopamine (DA) depletion causes motor deficits, the origin of the associated cognitive impairments remains a matter of debate. The present study aimed to decipher the influence of a partial 6-hydroxydopamine (6-OHDA) lesion of striatal DA nerve terminals in rats performing a reaction time task previously used to assess cognitive deficits in PD patients. The effects of two behavioral manipulations-foreperiod duration and stimulus-response congruence- known to affect motor processes and executive control, respectively, were studied over 8 weeks postsurgery in control and lesion animals.

Two weeks after surgery, the lesion abolished the effect of foreperiod, confirming the direct involvement of striatal DA in motor processes, but failed to alter the effect of congruence. During the following weeks, the effect of foreperiod was reinstated, indicating a recovery of lesion-induced motor symptoms. This recovery was accompanied by a progressive increase of the congruence effect, signaling an executive control deficit in lesion animals. This result provides the first evidence that 6-OHDA lesioned rats exhibit the same cognitive impairment as PD patients in this task. The deficit, however, built up progressively after the lesion and may result from adaptations mitigating lesion-induced motor deficits.

\section{Introduction}

Cognitive impairments associated with Parkinson's disease (PD) include deficits of executive functions, such as planning and working memory (Brown and Marsden, 1990; Owen, 2004), attention (Lewis et al., 2003) and decision making (Brand et al., 2004). While there is general agreement that impaired basal ganglia (BG) outputs to cortical structures reduce thalamocortical feedbacks and contribute to motor deficits (Alexander and Crutcher, 1990; Boraud et al., 2002; Morris et al., 2006), the origin of cognitive impairments remains under debate. This is because the progressive degeneration of dopaminergic (DA) neurons in the substantia nigra pars compacta is associated with the dysfunction of other neurotransmitter systems and the degeneration of the frontal lobes over the course of the disease (Nagano-Saito et al., 2008). Therefore, direct involvement of the DA system in cognitive impairments remains to be demonstrated.

The present study attempted to determine how striatal dopamine depletion contributes to cognitive impairments. We stud-

\footnotetext{
Received May 23, 2011; revised June 27, 2011; accepted July 1, 2011.

Author contributions: A.C., F.V., and T.H. designed research; A.C., J.H., F.V., N.T.-L., and M.A. performed research; A.C., B.B., N.T.-L., and T.H. analyzed data; B.B., M.A., and T.H. wrote the paper.

B.B. is supported by the European Research Council under the European Community's Seventh Framework Programme (FP7/2007-2013 Grant Agreement no. 241077). We thank Karen Davranche, Francesca Sargolini, and Bruno Poucet for fruitful discussions.

The authors declare no competing financial interests.

Correspondence should be addressed to Thierry Hasbroucq, Laboratoire de Neurobiologie de la Cognition, Université de Provence, Case C, 3 Place Victor Hugo, 13331 Marseille Cedex 3, France. E-mail: thierry.hasbroucq@univ-provence.fr.

DOI:10.1523/JNEUROSCI.2550-11.2011

Copyright $\odot 2011$ the authors $\quad 0270-6474 / 11 / 3111929-05 \$ 15.00 / 0$
}

ied the influence of experimental striatal DA depletion on motor and executive control in rats performing a reaction time (RT) task adapted from human protocols. Both rat (Brown and Robbins, 1991) and human RT performance (Näätänen, 1971) depend on the duration of the interval, termed "foreperiod," that separates a warning signal from the stimulus. Foreperiod duration affects motor cortical excitability (Davranche et al., 2007). PD (Jurkowski et al., 2005) and lesions of the rat nigrostriatal pathways (Brown and Robbins, 1991) alter this effect, confirming that BG dysfunctions have associated motor symptoms. The spatial correspondence between stimuli and responses affects performance in both humans and rats (Courtière et al., 2007): subjects perform better when the spatial position of the stimulus and response match (congruent association) than when the positions do not match (incongruent association). As incongruent associations require prepotent response tendencies to be overridden, congruence effects reflect executive control (Kornblum et al., 1990). PD patients display larger congruence effects than controls (Praamstra and Plat, 2001; Schmiedt-Fehr et al., 2007), which supports the notion that striatal DA depletion impairs executive control (Wylie et al., 2010).

This paper details the influence of a partial bilateral 6-hydroxydopamine (6-OHDA) lesion of striatal DA nerve terminals (Sauer and Oertel, 1994) on the effects of foreperiod and congruence in rats. The lesion was predicted to (1) cause motor deficits resulting in a reduced foreperiod effect, and (2) impair executive control and thus increase the effect of congruence. However, after partial lesions, the effect of foreperiod seems to recover rapidly after surgery (Turle-Lorenzo et al., 2006). We 
thus examined the influence of this recovery on the cognitive effect of congruence over 8 postoperative weeks.

Results show that the recovery of the foreperiod effect was accompanied by a cognitive control impairment that mimicked deficits encountered by PD patients.

\section{Materials and Methods}

Subjects. Eighteen male Long-Evans hooded rats, three months old and weighing 120-150 g at the start of training were kept at $85 \%$ of the free-feeding weight. Rats were 6 months old at the time of surgery. The experimental protocols applied conformed to the requirements of the European Community.

Apparatus. Experiments took place in operant chambers (Fig. 1). Each chamber had one retractable lever, located $7 \mathrm{~cm}$ above the floor in the middle of the front panel, and two lateral food wells positioned $12 \mathrm{~cm}$ to either side of the central lever. The wells were equipped with photocell beams and served as response operanda, each one being connected to a pellet dispenser which provided a reward. Two loudspeakers were mounted, one on either side of the chamber. The speakers produced a high-pitch sound at $12 \mathrm{kHz}$ in frequency and $56 \mathrm{~dB}$ SPL in intensity and a low-pitch sound at $3 \mathrm{kHz}$ in frequency and $67 \mathrm{~dB}$ SPL in intensity.

Task. The temporal organization of trial events in the experimental and learning phases of the experiment are depicted in Figure 1, $A$ and $B$, respectively. Nine subjects were conditioned to associate the high-pitch sound to a left-side response and the low-pitch sound to a right-side response, the other nine being conditioned to the opposite rule. After 2 months of "bilateral" training, the two different sounds were delivered from a single side of the cage, with the side being selected randomly. Overall, 47 sessions of training were run. Incorrect trials and RT plus movement times longer than $1.5 \mathrm{~s}$ were immediately followed by lever retraction, a time-out of $2 \mathrm{~s}$, and omission of reward. A daily session consisted of 144 stimulus presentations, regardless of the nature of the response given by the animal.

Design. After surgery, a 1 week recovery period was given. Animals were then tested again on the task, $5 \mathrm{~d}$ per week for 7 weeks. Individual trial signal pitch was varied randomly, producing a $50 \%$ chance of right or left response. Each day, half of the animals performed the task with a $1.5 \mathrm{~s}$ foreperiod and the other half with a $1 \mathrm{~s}$ foreperiod.

Surgery. Animals, under xylazine $(15 \mathrm{mg} / \mathrm{kg}$, i.p.) and ketamine ( $100 \mathrm{mg} / \mathrm{kg}$, i.p.) anesthesia, were secured in a stereotaxic apparatus with the tooth bar set $3 \mathrm{~mm}$ below the interaural line. 6-OHDA ( $4 \mu \mathrm{g} / \mu \mathrm{l} / \mathrm{side} ; n=9)$ or vehicle $(0.1 \%$ ascorbic acid, $n=9)$ solutions were infused in a volume of $3 \mu \mathrm{l} /$ side for $6 \mathrm{~min}(1 \mu \mathrm{l} / 3$ min) through injection needles (30 gauge) inserted bilaterally into the dorsal striatum and connected by a polyethylene tubing to a Hamilton syringe fitted to a microdrive pump. The coordinates (Paxinos and Watson, 1986) were taken from bregma: AP, $+0.2 \mathrm{~mm}$; L, $\pm 3.5 \mathrm{~mm}$; DV, $-4.8 \mathrm{~mm}$ from

B are represented by the arrows.

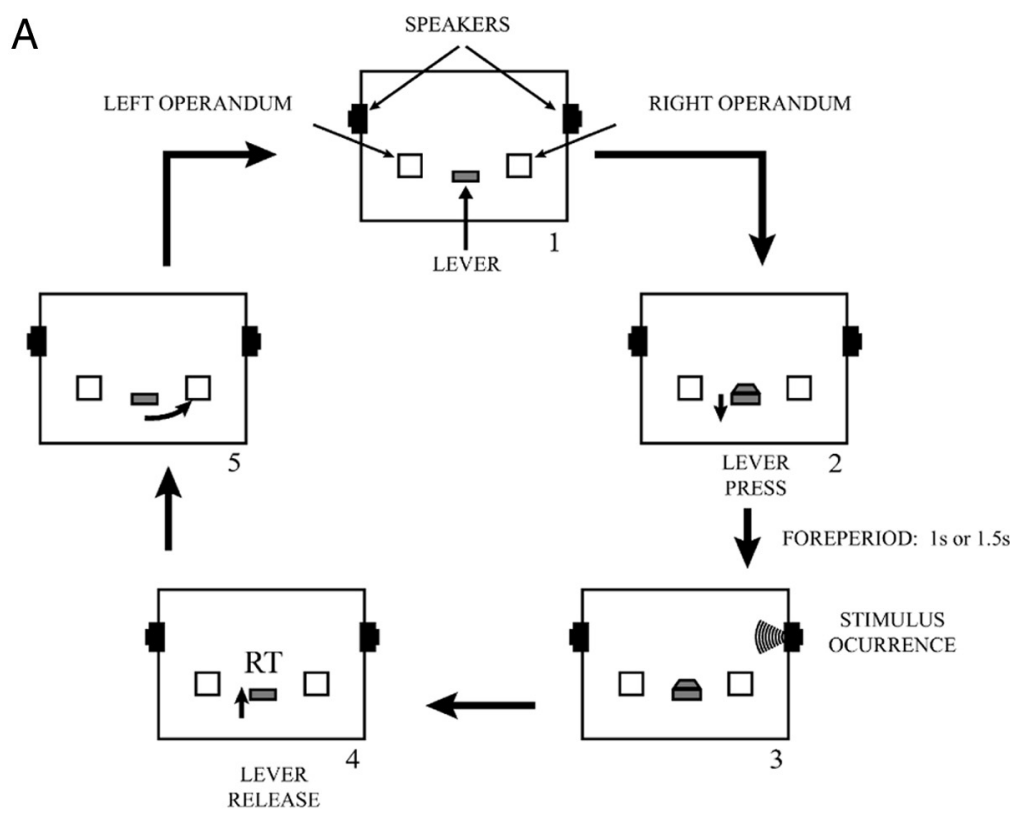

BILATERAL LEARNING
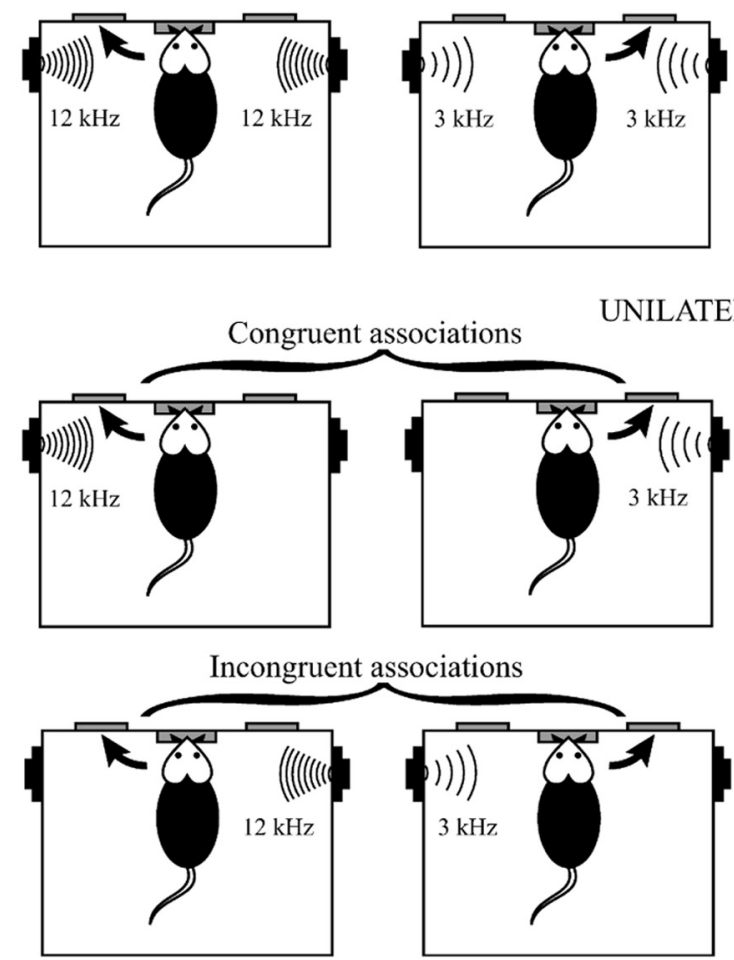

Figure 1. A, The main phases of a trial in the task. After an initial lever insertion into the chamber (phase 1) the subject was required to press the lever (phase 2) and to hold it down for either $1 \mathrm{~s}$ or a 1.5 s until the onset of a 3 or $12 \mathrm{kHz}$ sound delivered on a random side of the chamber for $300 \mathrm{~ms}$ (phase 3). The subject was to release the lever after the presentation of the sound, the delay between sound onset and lever release constituting the RT of the trial (phase 4). Visiting the response operandum corresponding to the learned rule of sound pitch-side constituted a correct response whereas visiting the opposite response operandum constituted a commission error. Correct responses were rewarded with the dispensing of a food pellet (phase 5). The next trial was initiated as soon as the subject depressed the lever. $\boldsymbol{B}$, Learning and testing phases of the experiment. During the learning phase, rats reacted to bilaterally presented sounds. During the experiment, they were to react to unilaterally presented sounds. The stimulus-response associations were congruent when the rat was to respond on the same side as the sound and incongruent when it was to respond on the side opposite to that of the sound. Correct responses

skull and from interaural zero; AP, $+9.2 \mathrm{~mm} ; \mathrm{L}, \pm 3.5 \mathrm{~mm}$; DV,+5.2 $\mathrm{mm}$, then averaged. Three animals did not recover from surgery. Postoperative testing was conducted with seven rats in the sham and eight subjects in the lesion group. 
Histological control of DA lesion: ${ }^{3} \mathrm{H}$-mazindol binding to dopamine uptake sites. Animals were anesthetized, their brains quickly removed after decapitation and frozen in dry ice, maintained at $-80^{\circ} \mathrm{C}$ until cryostat sectioning. Coronal $\left(10-\mu \mathrm{m}\right.$-thick) tissue sections were cut at $-20^{\circ} \mathrm{C}$ at the level of the striatum (between interaural coordinates AP, 11.04 and $8.04 \mathrm{~mm}$; based on Paxinos and Watson, 1986).

The loss of DA terminals in the striatum was indexed through the extent of DA denervation by analysis of ${ }^{3} \mathrm{H}$-mazindol binding to DA uptake sites, as described by Amalric et al. (1995). Autoradiograms were generated by apposing the sections to a ${ }^{3} \mathrm{H}$-sensitive screen for $21 \mathrm{~d}$ and were further quantified with a $\beta$ imager. Film autoradiograms were scanned and the extent of lesion and neuronal loss determined using a computer image analysis system. The extension of the 6-OHDA lesion was quantitatively analyzed between anteriority levels 10.68 and 9.12 $\mathrm{mm}$, according to the Paxinos and Watson (1986) atlas. These levels spanned the whole striatum except the more rostral regions including the nucleus accumbens, and the most caudal areas including the globus pallidus, which were not affected by the toxin. The extent of the lesion was determined by the loss of ${ }^{3} \mathrm{H}$-mazindol binding. Extent was expressed as a percentage of the lesioned area on the whole striatum, at each anteriority level for the right and the left hemisphere, and averaged for each hemisphere.

Data analysis. An ANOVA followed by a Student's $t$ test was used to compare the mean extent of striatal dopamine denervation of the lesion group at the rostrocaudal levels $(10.68-9.12 \mathrm{~mm})$.

The groups of subjects were analyzed week by week to obtain behavioral data. Analyzed variables were derived from the average of the five sessions performed in a weekly period, for each group in the different factor combinations. Commission errors, anticipations and omissions were expressed as a percentage of the total number of trials. The percentages were arcsine transformed before statistical analysis (Winer, 1971). The normality of RT data distributions were tested with the KolmogorovSmirnov test. Commission errors and RT were analyzed with overall ANOVAs for repeated measures; with group (control vs lesion) as a between-subject factor and time during presurgery and postsurgery (weeks), congruence and foreperiod as within-subject factors. Predicted differences across factor modalities were tested with the one-tailed Student's $t$ test. Anticipation and omission rates were submitted to similar ANOVAs. For concision, the results of these analyses, in line with the conclusions drawn from RT and error commission rate, are not reported but are available on request. For RT analysis, the trials corresponding to errors were discarded and the remaining correct responses averaged for the different combinations of factor modalities. We first compared the subjects' performance during the preoperative week. Then, to assess the acute effects of the lesion, we compared the subjects' performance during the preoperative week and during the first week after the recovery period (the second week after surgery). Finally, to assess the evolution of the effects induced by the lesion with postoperative time, we contrasted the subjects' performance during the 7 postoperative weeks of testing.

\section{Results}

\section{Histological detection of dopamine loss}

The loss of ${ }^{3} \mathrm{H}$-mazindol labeling extended upon the whole rostrocaudal levels of the striatum in 6-OHDA lesion subjects (Fig. 2). Intrastriatal 6-OHDA infusions produced bilateral lesions restricted to the dorsal striatum sparing the fibers located along the lateral ventricle. A mean $50 \pm 12.7 \%$ decrease of ${ }^{3} \mathrm{H}$-mazindol labeling was found along the striatal rostrocaudal axis. ${ }^{3} \mathrm{H}$-mazindol labeling within the core of the denervation was significantly different from labeling in the adjacent striatum $\left(F_{(1,7)}=177,19, p<0.01\right.$, Student's $t$ test, $p<0.01)$. The extent of the lesioned area did not differ across the five different anteriority levels (no interaction between lesion and anteriority $p>0.05$ ). These results are consistent with a loss of endogenous striatal DA contents as assessed by HPLC measurement after similar 6-OHDA treatment (Amalric et al., 1995).

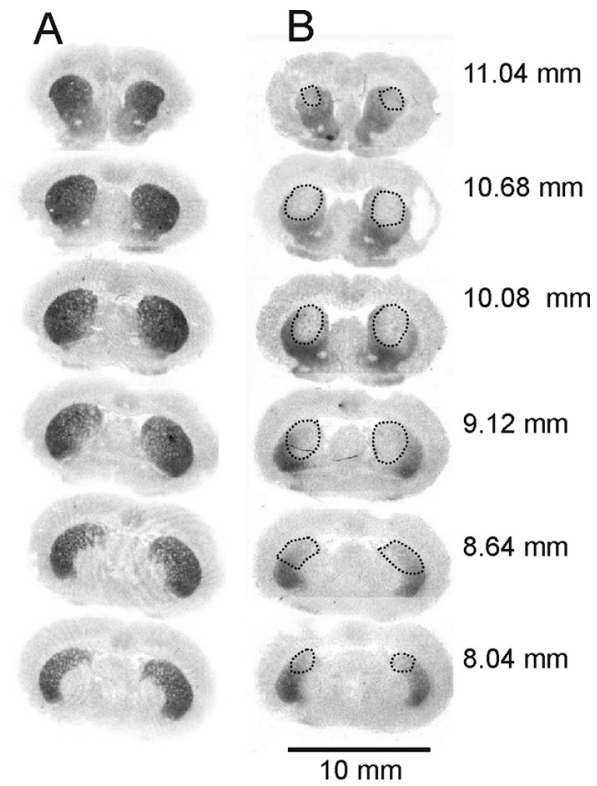

Figure 2. $\quad \boldsymbol{A}, \boldsymbol{B}$, Digitized autoradiographic images of frontal sections at striatal level (from 11.04 to $8.04 \mathrm{~mm}$ relative to interaural zero; Paxinos and Watson, 1986) illustrating ${ }^{3} \mathrm{H}$ mazindol binding in control $(\boldsymbol{A})$ and dopamine-lesioned $(\boldsymbol{B})$ representative subjects. The dotted lines delineate the dopamine denervated area. Scale bar, $10 \mathrm{~mm}$.

\section{Behavioral variables}

The distributions of RT data did not differ from normality in any condition of the design (all $p$ values $>0.20$ ). Lesion-induced effects were manifest on RT and error rate. The motor manipulation (foreperiod) affected RT while the executive control manipulation (congruence) affected the error rate. Behavioral results are presented in Figure 3.

\section{Preoperative performance}

Before surgery, the two groups were comparable in terms of performance. As expected, foreperiod and congruence affected motor and executive control processes, respectively.

Rats reacted faster for congruent than for incongruent associations, $F_{(1,13)}=44.58, p<0.01$ and were faster when the foreperiod was long than when it was short, $F_{(1,13)}=175.21, p<0.01$. No interaction reached significance level on this dependent variable (all $p$ values $>0.05$ ).

Rats committed more errors for incongruent than for congruent associations, $F_{(1,13)}=38.53, p<0.01$. No interaction reached significance level on this dependent variable (all $p$ values $>0.05$ ).

\section{Short-term effects}

During the second postoperative week, the lesion affected the rats' motor processes while leaving their executive control unaffected.

The lesion abolished the effect of foreperiod on RT (Fig. 3A). Rats displayed longer RTs post- than presurgery, $F_{(1,13)}=12.46$, $p<0.01$. Animals reacted faster for congruent than for incongruent associations, $F_{(1,13)}=109.62, p<0.01$, and for the long than for the short foreperiod, $F_{(1,13)}=87.55, p<0.01$. The effect of foreperiod was smaller in the lesion than in the control group (group $\times$ foreperiod interaction, $F_{(1,13)}=10.40, p<0.01$ ). The effect of foreperiod was decreased (and became nil) during the second postoperative week for lesion animals, $F_{(1,7)}=88.18, p<$ 0.01 , but not for sham operated rats, $F_{(1,6)}=1.03, p>0.05$ (three-term interaction: $F_{(1,13)}=20.93, p<0.01$ ). 
A
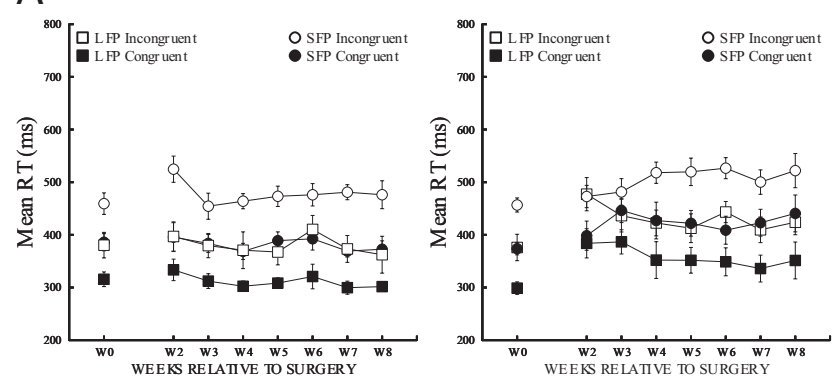

B

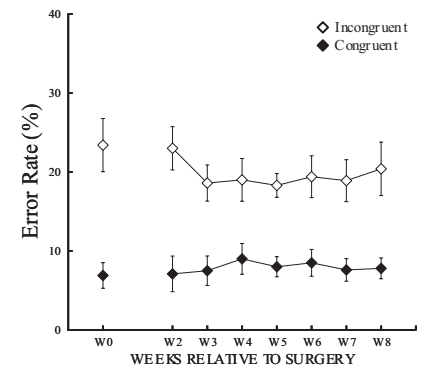

SHAM

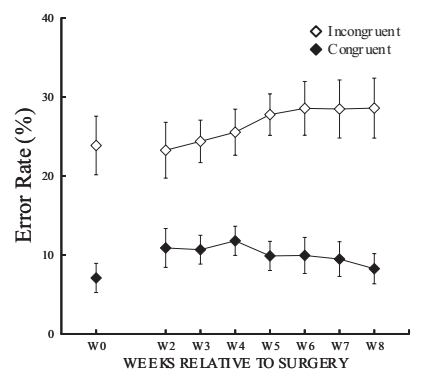

LESION

Figure 3. Behavioral results for control (left) and lesion animals (right) as a function of weeks relative to surgery (abcissae), with W, W- 0 denoting the preoperative week. $A$, Reaction time (ordinate) for congruent (circles) and incongruent (squares) associations and for short (empty symbols) and long (full symbols) foreperiods. B, Error rate for congruent (full diamonds) and incongruent (empty diamonds) associations. Since the analysis revealed no effect of foreperiod, neither as a main effect nor as a component term in an interaction, the points corresponding to the short and long foreperiods are averaged on the graphs. In all graphs, vertical bars indicate SES.

The analysis performed on the error rate failed to reveal an acute effect of the lesion on executive control (Fig. 3B). Lesion and control rats committed more errors for incongruent than for congruent associations, $F_{(1,13)}=44.09, p<0.01$. No other factor affected this dependent variable (all $p$ values $>0.05$ ).

\section{Long-term effects}

In lesion rats, the foreperiod effect on RT (nil after the lesion) gradually recovered the presurgical level at the fourth postoperative week. The effect of congruence on error commission rate progressively increased with postoperative weeks and became larger in lesion than in control animals, indicating an impairment of executive control in the former group. The recovery of lesioninduced motor deficits was thus accompanied by the development of an executive control impairment.

There was no main effect of week on RT, $F_{(6,78)}=1.29, p>0.05$. Rats reacted faster for the long foreperiod than for the short, $F_{(1,13)}=$ 158.60, $p<0.01$, and for congruent than for incongruent associations, $F_{(1,13)}=126.72, p<0.01$. Overall, the foreperiod effect increased across postoperative weeks (week $\times$ foreperiod interaction, $\left.F_{(6,78)}=2.80 ; p<0.025\right)$. However, this increase was present for lesion animals, $F_{(6,42)}=5.16, p<0.01$, but not for controls, $F_{(6,36)}<1$ (group $\times$ foreperiod $\times$ congruence interaction: $F_{(6,78)}=$ $3.08, p<0.01$ ). To delineate the time course of this effect, the analysis was restricted to the third to the seventh postoperative weeks. The foreperiod effect was stable during the postoperative weeks (week $X$ foreperiod interaction, $F_{(5,65)}=1.80, p>0.05$ ), and this was true for the two groups (group $\times$ week $\times$ foreperiod interaction, $F_{(5,65)}=$ $1.09, p>0.05)$. This indicates that the effect of foreperiod was rein-

stated in lesion animals as early as the third postoperative week, suggesting a relatively fast recovery of motor processes.

Rats committed more errors for incongruent than for congruent associations, $F_{(1,13)}=62.03, p<0.01$. The effect of congruence increased across postoperative weeks for lesion, $F_{(6,42)}=2.49, p<$ 0.05 , but not for controls, $F_{(6,36)}=1.27, p>0.05$ (group $\times$ week $\times$ compatibility interaction, $\left.F_{(6,78)}=2.36, p<0.05\right)$. As expected on the basis of PD patients' performance, contrast comparisons revealed that for the eighth postoperative week, the effect of congruence was larger in the lesion that in the control group (one-tailed Student's $t$ test $=1.995, p<0.05$ ). Eight weeks after the surgery, lesion rats thus experienced an impairment of executive control.

\section{Discussion}

Two weeks after surgery, the lesion drastically impaired motor readiness. During the following postoperative weeks, this acute effect vanished and the difference in performance between incongruent and congruent associations progressively increased, resulting in a larger congruence effect for lesion animals than for sham operated ones.

Before the lesion, rats reacted faster for the long foreperiod (1.5 s) than for the short foreperiod (1 s), confirming that they attained a higher level of motor readiness in the former than in the latter condition (Brown and Robbins, 1991). Futhermore, rats reacted faster and committed fewer errors for congruent than for incongruent associations. This effect reveals a response conflict originating from the parallel processing of the irrelevant (tone location) and relevant (tone pitch) information conveyed by the stimulus (Kornblum et al., 1990). These parallel routes correspond to different modes of action control (Balleine and O'Doherty, 2010). The route that conveys the relevant information is controlled and relatively slow, while the alternative is automatic and fast. The fast and slow routes converge onto cortical areas where they activate neural populations involved in response implementation (Kornblum et al., 1990). When the stimulusresponse association is congruent, the fast route activates the required response and facilitates its implementation. In contrast, when the stimulus-response association is incongruent, the fast route activates the nonrequired response. This activation must then be overridden, engaging executive control processes and impairing the implementation of the required response. The net congruence effect thus reflects the balance between the activation of responses along the fast and slow route, respectively. The demonstration of such an effect in rats shows that these rodents, like humans, experience and solve a response conflict when making a decision in the same task.

Two weeks after the surgery, the effect of foreperiod became nil in lesion rats. In line with previous results (Brown and Robbins, 1991), this acute effect suggests that the lesion reduced the excitability of the cortex by increasing inhibitory GABAergic outputs of the BG to the thalamus, thereby inducing motor symptoms (Alexander and Crutcher, 1990). The lesion-induced reduction in BG output likely affected cortical excitability in such a way that the animals were no longer able to efficiently prepare their responses when the foreperiod was long (Brown and Robbins, 1991; Amalric et al., 1995; Meck, 1996). The lesion did not affect the congruence effect, which suggests that during the second postoperative week, the animal's ability to resolve response conflict was unaltered. This may be because the lesion reduced the activations triggered via the fast and slow routes by a comparable amount. In other words, the balance between the two sources of response activation was preserved. This suggests that, in contrast to lesion-induced motor symptoms, the increased 
congruence effect in PD patients may not be an immediate (acute) effect of nigrostriatal DA depletion but rather may reflect compensatory adaptations.

By studying performance during eight postsurgical weeks, we followed the time course of this functional recovery. The present data show that a partially lesioned nigrostriatal system can recover within 3 weeks, which restores motor readiness. This rapid recovery suggests that another mechanism can compensate for the acute deficits caused by DA depletion. Examination of the error commission rate sheds light on this compensation mechanism. In lesion rats, the congruence effect on error commission rate increased with postoperative week and was larger 8 weeks after the surgery than it was at the same period in control animals. Such an increase reveals that the (automatic) response activation by the (irrelevant) stimulus position became more important as time elapsed from the surgery. This could be due to the fact that the motor cortex became progressively more responsive to the irrelevant stimulus location. Indeed, the relative strength of the location-based (over pitch based) processing grew while the lesioninduced motor symptoms recovered. The increase of the congruence effect could thus be a functional counterpart of the compensation of motor symptoms (for related interpretations, see Redgrave et al., 2010). A contribution of the frontal cortex, a structure that mediates action selection and temporal preparation possibly through its action on the striatum (Risterucci et al., 2003; Narayanan and Laubach, 2006; Kimchi and Laubach, 2009), to the articulation of executive control and motor readiness can be conjectured. This issue should be addressed in future work.

To summarize, by progressively reducing the animals' ability to resolve response competition in the Simon task (Simon, 1990), the lesion mimicked one executive control deficit encountered by PD patients (Schmiedt-Fehr et al., 2007). This finding must be related to results obtained in rats with tasks engaging executive processes for interval timing and temporal control (Coull et al., 2010).

\section{References}

Alexander GE, Crutcher MD (1990) Functional architecture of basal ganglia circuits: neural substrates of parallel processing. Trends Neurosci 13:266-271.

Amalric M, Moukhles H, Nieoullon A, Daszuta A (1995) Complex deficits on reaction time performance following bilateral intrastriatal 6-OHDA infusion in the rat. Eur J Neurosci 7:972-980.

Balleine BW, O’Doherty JP (2010) Human and rodent homologies in action control: corticostriatal determinants of goal-directed and habitual action. Neuropsychopharmacology 35:48-69.

Boraud T, Bezard E, Bioulac B, Gross CE (2002) From single extracellular unit recording in experimental and human Parkinsonism to the development of a functional concept of the role played by the basal ganglia in motor control. Prog Neurobiol 66:265-283.

Brand M, Labudda K, Kalbe E, Hilker R, Emmans D, Fuchs G, Kessler J, Markowitsch HJ (2004) Decision-making impairments in patients with Parkinson's disease. Behav Neurol 15:77-85.

Brown RG, Marsden CD (1990) Cognitive function in Parkinson's disease: from description to theory. Trends Neurosci 13:21-29.

Brown VJ, Robbins TW (1991) Simple and choice reaction time performance following unilateral striatal depletion in the rat. Brain 114:513-525.

Coull JT, Cheng RK, Meck WH (2010) Neuroanatomical and neurochemical substrates of timing. Neuropsychopharmacology 36:3-25.
Courtière A, Hardouin J, Burle B, Vidal F, Hasbroucq T (2007) The Simon effect in the rat: a new model for studying the neural bases of the dualroute architecture. Behav Brain Res 179:69-75.

Davranche K, Tandonnet C, Burle B, Meynier C, Vidal F, Hasbroucq T (2007) The dual nature of time preparation: neural activation and suppression revealed by transcranial magnetic stimulation of the motor cortex. Eur J Neurosci 25:3766-3774.

Jurkowski AJ, Stepp E, Hackley SA (2005) Variable foreperiod deficits in Parkinson's disease: dissociation across reflexive and voluntary behaviors. Brain Cogn 58:49-61.

Kimchi EY, Laubach M (2009) Dynamic encoding of action selection by the ventral striatum. J Neurosci 29:3148-3159.

Kornblum S, Hasbroucq T, Osman A (1990) Dimensional overlap; cognitive basis for stimulus-response compatibility—a model and taxonomy. Psychol Rev 97:253-270.

Lewis SJ, Dove A, Robbins TW, Barker RA, Owen AM (2003) Cognitive impairments in early Parkinson's disease are accompanied by reductions in activity in frontostriatal neural circuitry. J Neurosci 23:6351-6356.

Meck WH (1996) Neuropharmacology of timing and time perception. Cogn Brain Res 3:227-242.

Morris G, Nevet A, Arkadir D, Vaadia E, Bergman H (2006) Midbrain dopamine neurons encode decisions for future action. Nat Neurosci 9:1057-1063.

Näätänen R (1971) Non-aging fore-periods and simple reaction time. Acta Psychol 35:316-327.

Nagano-Saito A, Leyton M, Monchi O, Goldberg YK, He Y, Dagher A (2008) Dopamine depletion impairs frontostriatal functional connectivity during a set-shifting task. J Neurosci 28:3697-3706.

Narayanan NS, Laubach M (2006) Top-down control of motor cortex ensembles by dorsomedial prefrontal cortex. Neuron 52:921-931.

Owen AM (2004) Cognitive dysfunction in Parkinson's disease: the role of frontostriatal circuitry. Neuroscientist 10:525-537.

Paxinos G, Watson C (1986) The rat brain in stereotaxic coordinates, Ed 2. New York: Academic.

Praamstra P, Plat FM (2001) Failed suppression of direct visuomotor activation in Parkinson's disease. J Cogn Neurosci 13:31-43.

Redgrave P, Rodriguez M, Smith Y, Rodriguez-Oroz MC, Lehericy S, Bergman H, Agid Y, DeLong MR, Obeso JA (2010) Goal-directed and habitual control in the basal ganglia: implications for Parkinson's disease. Nat Rev Neurosci 11:760-772.

Risterucci C, Terramorsi D, Nieoullon A, Amalric M (2003) Excitotoxic lesions of the prelimbic-infralimbic areas of the rodent prefrontal cortex disrupt motor preparatory processes. Eur J Neurosci 17:1498-1508.

Sauer H, Oertel WH (1994) Progressive degeneration of nigrostriatal dopamine neurons following intrastriatal terminal lesions with 6-hydroxydopamine: combined retrograde tracing and immunocytochemical study in the rat. Neuroscience 59:401-415.

Schmiedt-Fehr C, Schwendemann G, Herrmann M, Basar-Eroglu C (2007) Parkinson's disease and age-related alterations in brain oscillations during a Simon task. Neuroreport 18:277-281.

Simon JR (1990) The effect of an irrelevant directional cue on human information processing. In: Stimulus-response compatibility: an integrated perspective (Proctor RW, Reeve TG, eds), pp 31-88. Amsterdam: North-Holland.

Turle-Lorenzo N, Maurin B, Puma C, Chezaubernard C, Morain P, Baunez C, Nieoullon A, Amalric M (2006) The dopamine agonist piribedil with L-dopa improves attentional dysfunction: relevance for Parkinson's disease. J Pharmacol Exp Ther 319:914-923.

Winer BJ (1971) Statistical principles in experimental design. New York: McGraw-Hill.

Wylie SA, Ridderinkhof KR, Bashore TR, van den Wildenberg WP (2010) The effect of Parkinson's disease on the dynamics of online and proactive cognitive control during action selection. J Cogn Neurosci 22:2058-2073. 